\title{
Kleene algebras with implication
}

\author{
José Luis Castiglioni, Sergio Arturo Celani, And Hernán Javier \\ SAN MARTÍN
}

\begin{abstract}
Inspired by an old construction due to J. Kalman that relates distributive lattices and centered Kleene algebras, in this paper we study an equivalence for certain categories whose objects are algebras with implication $(H, \wedge, \vee, \rightarrow, 0,1)$ which satisfy the following property for every $a, b, c \in H$ : if $a \leq b \rightarrow c$, then $a \wedge b \leq c$.
\end{abstract}

\section{Introduction}

Motivated by results due to Kalman [11], R. Cignoli in [8] proved that the construction of [11] induces a functor $\mathrm{K}$ from the category of bounded distributive lattices into the category of centered Kleene algebras. It was also shown in [8] that $\mathrm{K}$ has a left adjoint [8, Theorem 1.7]. He also determined an equivalence between the category of bounded distributive lattices and a full subcategory of centered Kleene algebras [8, Theorem 2.4]. In particular, there exists an equivalence between the category of Heyting algebras and the category of centered Nelson algebras [8, Theorem 3.14]. Later, these results were extended in $[4,5]$ in the context of residuated lattices.

A possible generalization of Heyting algebras is provided by the notion of algebras with implication, $D L I$-algebras for short [6]. The question naturally arises whether it is possible to consider some subcategory of $D L I$-algebras and some category of centered Kleene algebras with implication in order to obtain an equivalence between them, making the following diagram commute,

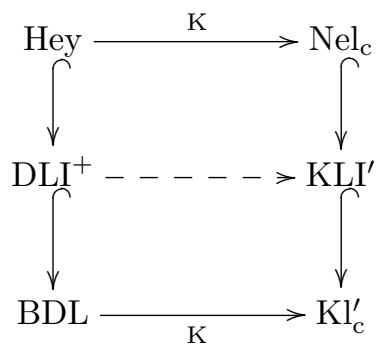

Presented by C. Tsinakis.

Received October 14, 2015; accepted in final form March 25, 2016.

2010 Mathematics Subject Classification: Primary: 06D99; Secondary: 06D05, 08A30, $08 \mathrm{~A} 62$.

Key words and phrases: involutive distributive lattices, centered Kleene algebras, lattices with implication.

This work was supported by grants CONICET PIP 112-201101-00636 and UNLP 11/X667. 
In this article, we answer this question in the positive. We consider $D L I$ algebras $(H, \wedge, \vee, \rightarrow, 0,1)$ which also satisfy the following property: for every $a, b, c \in H$, if $a \leq b \rightarrow c$, then $a \wedge b \leq c$; or equivalently, the inequality $a \wedge(a \rightarrow b) \leq b$, for every $a, b \in H$.

The applications of Kalman's construction given in [8], together with the interest in $D L I$-algebras [6], suggest that it is potentially fruitful to understand Kalman's work in the context of $D L I$-algebras. We do this in the present paper.

The paper is organized as follows. In Section 2, we present some results that we use later about Kalman's functor for bounded distributive lattices and Heyting algebras. In Section 3, we introduce certain varieties of $D L I$-algebras and we study the relation among them. In Section 4, we build up categorical equivalences for the mentioned varieties extending the original Kalman construction. In Section 5, we establish an order isomorphism for the lattice of congruences of any $D L I$-algebra with the additional inequality $a \wedge(a \rightarrow b) \leq b$. In Section 6, we make some final remarks.

All the categories considered in this paper have an underlying class of algebras, so we shall use the same notation in both cases.

\section{Basic results}

In the process of our research on this topic, we have found it useful to place our problems in the following general context.

We assume the reader is familiar with bounded distributive lattices and Heyting algebras [1]. A De Morgan algebra is an algebra $(H, \wedge, \vee, \sim, 0,1)$ of type $(2,2,1,0,0)$ such that $(H, \vee, \wedge, 0,1)$ is a bounded distributive lattice and $\sim$ fulfills the equations $\sim \sim x=x$ and $\sim(x \vee y)=\sim x \wedge \sim y$. An operation $\sim$ which satisfies the previous two equations is called a involution. A Kleene algebra is a De Morgan algebra in which the inequality $x \wedge \sim x \leq y \vee \sim y$ holds. A centered Kleene algebra is a Kleene algebra with an element c such that $\mathrm{c}=\sim \mathrm{c}$. It follows from the distributivity of the lattice that $\mathrm{c}$ is necessarily unique. We write BDL for the category of bounded distributive lattices and $\mathrm{Kl}_{\mathrm{C}}$ for the category of centered Kleene algebras.

Remark 2.1. If $H$ is a bounded distributive lattice, we define

$$
\mathrm{K}(H):=\{(a, b) \in H \times H: a \wedge b=0\} .
$$

We have that $(\mathrm{K}(H), \vee, \wedge, \mathrm{c}, \sim, 0,1)$ is a centered Kleene algebra by defining the following operations:

$$
\begin{gathered}
(a, b) \vee(d, e):=(a \vee d, b \wedge e), \quad(a, b) \wedge(d, e):=(a \wedge d, b \vee e), \\
\sim(a, b):=(b, a), \quad 0:=(0,1), \quad 1:=(1,0), \quad c:=(0,0) .
\end{gathered}
$$

Moreover, if $f: H \rightarrow G$ is a morphism in BDL, then $\mathrm{K}(f): \mathrm{K}(H) \rightarrow \mathrm{K}(G)$ given by $\mathrm{K}(f)(x, y)=(f(x), f(y))$ is a morphism in $\mathrm{Kl}_{\mathrm{c}}$. Then there is a functor $\mathrm{K}$ from $\mathrm{BDL}$ to $\mathrm{Kl}_{\mathrm{c}}$. 
Applying an extension of Priestley duality, in [8] Cignoli proves that K has a left adjoint. He also proves that there exists a categorical equivalence between BDL and a full subcategory of $\mathrm{Kl}_{\mathrm{c}}$ in which the objects satisfy a condition in its associated Priestley spaces.

Let $(T, \vee, \wedge, \sim, \mathrm{c}, 0,1)$ be a centered Kleene algebra. We define

$$
\mathrm{C}(T):=\{x \in T: x \geq \mathrm{c}\} .
$$

We have that $(\mathrm{C}(T), \wedge, \vee, \mathrm{c}, 1)$ is a bounded distributive lattice. Moreover, if $g: T \rightarrow U$ is a morphism in $\mathrm{Kl}_{\mathrm{c}}$ then $\mathrm{C}(g): \mathrm{C}(T) \rightarrow \mathrm{C}(U)$ given by $\mathrm{C}(g)(x)=$ $g(x)$ is a morphism in BDL. Then we have a functor $\mathrm{C}$ from $\mathrm{Kl}_{\mathrm{c}}$ to BDL. See $[4,5,8]$.

Remark 2.2. If $H$ is in BDL, then the map $\alpha_{H}: H \rightarrow \mathrm{C}(\mathrm{K}(H))$ given by $\alpha_{H}(x)=(x, 0)$ is an isomorphism in BDL. If $T \in \mathrm{Kl}_{\mathrm{c}}$, then $\beta_{T}: T \rightarrow \mathrm{K}(\mathrm{C}(T))$ given by $\beta_{T}(x)=(x \vee \mathrm{c}, \sim x \vee \mathrm{c})$ is an injective map which is a morphism in $\mathrm{Kl}_{\mathrm{c}}$. That $\beta_{T}$ is well defined follows since in any Kleene algebra, we have that $x \wedge \sim x \leq \mathrm{c}$, so $(x \vee \mathrm{c}) \wedge(\sim x \vee \mathrm{c})=(x \wedge \sim x) \vee \mathrm{c}=\mathrm{c}$.

Theorem 2.3. With the notation above, we have that the functor $\mathrm{K}$ is right adjoint of $\mathrm{C}$.

Proof. This follows from [4, Theorem 3.6]. See also [8, Theorem 1.7].

Let $T \in \mathrm{Kl}_{\mathrm{c}}$. We consider the following algebraic condition:

(CK) For every $x, y \geq \mathrm{c}$ such that $x \wedge y \leq \mathrm{c}$, there exists $z$ such that $z \vee \mathrm{c}=x$ and $\sim z \vee \mathrm{c}=y$.

Remark 2.4. Let $H \in \mathrm{BDL}$. Given two elements $x, y$ in $\mathrm{K}(H)$ such that $x, y \geq \mathrm{c}$ and $x \wedge y \leq \mathrm{c}$, they are of the form $x=(a, 0)$ and $y=(b, 0)$, with $a \wedge b=0$. The element $z=(a, b)$ in $\mathrm{K}(H)$ satisfies $z \vee \mathrm{c}=x$ and $\sim z \vee \mathrm{c}=y$. Therefore, $\mathrm{K}(H)$ satisfies $(\mathrm{CK})$.

The condition (CK) is not necessarily satisfied in every centered Kleene algebra, as we show in the following example.

Example 2.5. Consider the centered Kleene algebra of Figure 1. We have that $x, y \geq \mathrm{c}$ and $x \wedge y=\mathrm{c}$. However, there is no $z$ such that $z \vee \mathrm{c}=x$ and $\sim z \vee \mathrm{c}=y$.

We write $\mathrm{Kl}_{\mathrm{c}}^{\prime}$ for the full subcategory of $\mathrm{Kl}_{\mathrm{c}}$ whose objects satisfy the condition $(\mathrm{CK})$. For $T \in \mathrm{Kl}_{\mathrm{c}}$, the following remark follows from the definition of $\beta_{T}$.

Remark 2.6. $T$ satisfies (CK) if and only if $\beta_{T}$ is a surjective map.

Straightforward computations based on previous results of this section prove the following result.

Theorem 2.7. The functors $\mathrm{K}$ and $\mathrm{C}$ establish a categorical equivalence between $\mathrm{BDL}$ and $\mathrm{Kl}_{\mathrm{c}}^{\prime}$ with natural isomorphisms $\alpha$ and $\beta$. 


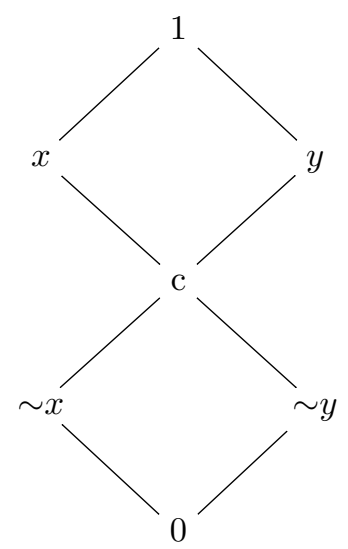

FiguRE 1. An example of a centered Kleene algebra which does not satisfy condition $(\mathrm{CK})$.

Remark 2.8. In [8, Theorem 2.4], it was proved that there exists a categorical equivalence between BDL and the full subcategory of $\mathrm{Kl}_{\mathrm{c}}$ whose objects satisfy a topological condition called the interpolation property. It was observed by M. Sagastume [12] that if $T \in \mathrm{Kl}_{\mathrm{c}}$, then $T$ satisfies the interpolation property if and only if $T$ satisfies (CK). Moreover, $\mathrm{Kl}_{\mathrm{c}}^{\prime}$ is the full subcategory of $\mathrm{Kl}_{\mathrm{c}}$ whose objects satisfy the interpolation property. This result was never published. For completeness, we give a direct proof of it for the interested reader at the end of this article.

A quasi-Nelson algebra is a Kleene algebra such that for each pair $x, y$, there exists $x \rightarrow(\sim x \vee y)$, where $\rightarrow$ is the Heyting implication. We define the binary operation $\Rightarrow$ as $x \Rightarrow y=x \rightarrow(\sim x \vee y)$. A Nelson algebra is a quasi-Nelson algebra satisfying the equation $(x \wedge y) \Rightarrow z=x \Rightarrow(y \Rightarrow z)$. A quasi-Nelson algebra is a Nelson algebra if and only if it satisfies the interpolation property [8, Theorem 3.5].

Let $(H, \vee, \wedge, \rightarrow, 0,1)$ be a Heyting algebra. We consider the following binary operation in $\mathrm{K}(H)$ (see $[10,14])$ :

$$
(a, b) \Rightarrow(d, e):=(a \rightarrow d, a \wedge e) .
$$

The algebra $(K(H), \wedge, \vee, \Rightarrow, \mathrm{c}, 0,1)$ is a centered Nelson algebra. We write Hey for the category of Heyting algebras and $\mathrm{Nel}_{\mathrm{c}}$ for the category of centered Nelson algebras. For the following result, see [4, Proposition 3.7] and [8, Theorem 3.14].

Theorem 2.9. Kalman's adjunction restricts to an adjoint equivalence:

$$
\mathrm{K} \dashv \mathrm{C}: \mathrm{Hey} \rightarrow \mathrm{Nel}_{\mathrm{c}} \text {. }
$$

An involutive residuated lattice is a bounded, integral and commutative residuated lattice $(T, \vee, \wedge, *, \rightarrow, 0,1)$ such that for every $x \in T$, we have that 
$\neg \neg x=x$, where $\neg x:=x \rightarrow 0$ and 0 is the least element of $T$ [3]. In an involutive residuated lattice, we have

$$
x * y=\neg(x \rightarrow \neg y) \quad \text { and } \quad x \rightarrow y=\neg(x * \neg y) .
$$

A Nelson lattice [3] is an involutive residuated lattice $(T, \vee, \wedge, *, \rightarrow, 0,1)$ which satisfies the additional inequality $\left(x^{2} \rightarrow y\right) \wedge\left((\neg y)^{2} \rightarrow \neg x\right) \leq x \rightarrow y$, where $x^{2}:=x * x$. See also [13].

Let $(T, \vee, \wedge, \Rightarrow, \sim, 0,1)$ be a Nelson algebra. It follows from [3, Theorem 3.1] that $(T, \wedge, \vee, *, \rightarrow, 0,1)$ is a Nelson lattice by defining

$$
\begin{aligned}
x * y & :=\sim(x \Rightarrow \sim y) \vee \sim(y \Rightarrow \sim x), \\
x \rightarrow y & :=(x \Rightarrow y) \wedge(\sim y \Rightarrow \sim x) .
\end{aligned}
$$

Moreover, $\neg x=x \rightarrow 0=\sim x$.

Let $(T, \wedge, \vee, *, \rightarrow, 0,1)$ be a Nelson lattice. It follows from [3, Theorem 3.6] that $(T, \vee, \wedge, \Rightarrow, \sim, 0,1)$ is a Nelson algebra by defining

$$
x \Rightarrow y:=x^{2} \rightarrow y \quad \text { and } \quad \sim x:=\neg x .
$$

Moreover, it can be proved that centered Nelson algebras and Nelson lattices are equationally equivalent (see [3, Theorem 3.11]).

Remark 2.10. Let $(H, \vee, \wedge, \rightarrow, 0,1)$ be a Heyting algebra. For $(a, b)$ and $(d, e)$ in $\mathrm{K}(H)$, the operations $*$ and $\rightarrow$ defined previously take the form

$$
\begin{aligned}
(a, b) *(d, e) & =(a \wedge d,(a \rightarrow e) \wedge(d \rightarrow b)), \\
(a, b) \rightarrow(d, e) & =((a \rightarrow d) \wedge(e \rightarrow b), a \wedge e) .
\end{aligned}
$$

We write $\rightarrow$ both for the implication in $H$ as for the implication in $\mathrm{K}(H)$.

A centered Nelson lattice is an algebra $(T, \vee, \wedge, *, \rightarrow, \mathrm{c}, 0,1)$, where the reduct $(T, \vee, \wedge, *, \rightarrow, 0,1)$ is a Nelson lattice and $\mathrm{c}$ is a constant such that $\neg \mathrm{c}=\mathrm{c}$.

Corollary 2.11. There exists an equivalence between Hey and the category of centered Nelson lattices.

\section{Some varieties of DLI-algebras}

Recall from [6] that an algebra $(H, \wedge, \vee, \rightarrow, 0,1)$ of type $(2,2,2,0,0)$ is a $D L I$-algebra if $(H, \wedge, \vee, 0,1)$ is a bounded distributive lattice and the following conditions are satisfied:

(I1) $(a \rightarrow b) \wedge(a \rightarrow d)=a \rightarrow(b \wedge d)$,

(I2) $(a \rightarrow d) \wedge(b \rightarrow d)=(a \vee b) \rightarrow d$,

(I3) $0 \rightarrow a=1$,

(I4) $a \rightarrow 1=1$.

Remark 3.1. Let $H$ be a $D L I$-algebra. It follows from (I1) that if $a \leq b$, then $d \rightarrow a \leq d \rightarrow b$, and it follows from (I2) that if $a \leq b$, then $b \rightarrow d \leq a \rightarrow d$. 
We write $\mathrm{DLI}^{+}$for the variety of $D L I$-algebras whose algebras satisfy the following equation:

(I5) $a \wedge(a \rightarrow b) \leq b$.

Remark 3.2. In any $D L I$-algebra the equation (I5) is equivalent to the following condition: for every $a, b, d$, if $a \leq b \rightarrow d$, then $a \wedge b \leq d$.

In every bounded distributive lattice $H$, if we define a binary operation $\rightarrow$ by $a \rightarrow b=1$ for every $a, b$, then $(H, \rightarrow)$ is is a $D L I$-algebra [7]. Taking into account this property, we can show that $\mathrm{DLI}^{+}$is a proper subvariety of the variety of $D L I$-algebras. In order to prove this, consider the chain of two elements $H_{2}=\{0,1\}$ with the above mentioned implication. We have that $1 \wedge(1 \rightarrow 0)=1 \not \leq 0$. Hence, $\mathrm{DLI}^{+}$is a proper subvariety of the variety of $D L I$-algebras. Furthermore, in every bounded distributive lattice $H$, it is possible to define a binary operation with the property that $(H, \rightarrow) \in \mathrm{DLI}^{+}$ as we show in the following example.

Example 3.3. Let $H$ be a bounded distributive lattice. Then $(H, \rightarrow) \in \mathrm{DLI}^{+}$ by defining

$$
a \rightarrow b= \begin{cases}1, & \text { if } a=0 \\ b, & \text { if } a \neq 0\end{cases}
$$

Let $H \in \mathrm{DLI}^{+}$. We consider the following equation:

(I6) $a \rightarrow a=1$.

We write $\mathrm{DLI}_{1}^{+}$for the subvariety of $\mathrm{DLI}^{+}$whose objects satisfy (I6).

Example 3.4. Consider the chain of three elements $H_{3}=\{0, a, 1\}$ such that $0<a<1$. We have that $\left(H_{3}, \rightarrow\right) \in \mathrm{DLI}^{+}$, where $\rightarrow$ is the binary operation given in Example 3.3. Since $a \rightarrow a=a \neq 1$, then $\left(H_{3}, \rightarrow\right) \notin \mathrm{DLI}_{1}^{+}$. Hence, we have that $\mathrm{DLI}_{1}^{+}$is a proper subvariety of $\mathrm{DLI}^{+}$.

Let $H \in \mathrm{DLI}_{1}^{+}$. Consider the following inequality:

(I7) $(a \rightarrow b) \wedge(b \rightarrow d) \leq a \rightarrow d$.

A weak Heyting algebra, or WH-algebra [2,7], is an ordered algebraic structure $(H, \wedge, \vee, \rightarrow, 0,1)$, where the reduct $(H, \wedge, \vee, 0,1)$ is a bounded distributive lattice and $\rightarrow$ is a binary operation on $A$ satisfying for all $a, b, c \in H$ the following conditions: $(a \rightarrow b) \wedge(a \rightarrow d)=a \rightarrow(b \wedge d),(a \rightarrow d) \wedge(b \rightarrow d)=(a \vee b) \rightarrow d$, $(a \rightarrow b) \wedge(b \rightarrow d) \leq a \rightarrow d$, and $a \rightarrow a=1$.

Some examples of $W H$-algebras that appear in the literature are the $R W H$-algebras [7] and the subresiduated lattices introduced by G. Epstein and A. Horn in [9]; these last structures were introduced as a generalization of Heyting algebras. A $R W H$-algebra is a $W H$-algebra that in addition satisfies the inequality (I5). A subresiduated lattice is a $R W H$-algebra that in addition satisfies the inequality

(I8) $a \rightarrow b \leq d \rightarrow(a \rightarrow b)$. 
We write RWH for the variety of $R W H$-algebras, and SRL for the variety of subresiduated lattices. Note that RWH is the subvariety of $\mathrm{DLI}_{1}^{+}$whose algebras satisfy (I7), and SRL is the subvariety of $\mathrm{DLI}^{+}$whose algebras satisfy (I7) and (I8).

Example 3.5. Consider the chain of four elements $H_{4}=\{0, a, b, 1\}$ with $0<a<b<1$. We consider the following binary operation:

\begin{tabular}{c|cccc}
$\rightarrow$ & 0 & $a$ & $b$ & 1 \\
\hline 0 & 1 & 1 & 1 & 1 \\
$a$ & 0 & 1 & 1 & 1 \\
$b$ & 0 & $a$ & 1 & 1 \\
1 & 0 & 0 & $b$ & 1
\end{tabular}

Straightforward computations show that $\left(H_{4}, \rightarrow\right) \in \mathrm{DLI}_{1}^{+}$. However, we have that $1 \rightarrow b=b$ and $b \rightarrow a=a$, so $(1 \rightarrow b) \wedge(b \rightarrow a)=a \not \leq 0=1 \rightarrow a$. Hence, RWH is a proper subvariety of $\mathrm{DLI}_{1}^{+}$.

Let $H \in \mathrm{DLI}^{+}$. We consider the following condition:

(A) For every $a, b$, if $a \wedge b=0$, then $a \leq b \rightarrow 0$.

We write $\mathrm{DLI}_{\neg}^{+}$for the class of algebras of $\mathrm{DLI}^{+}$whose algebras satisfy the condition (A). It is immediate that in every algebra of $\mathrm{DLI}_{\neg}^{+}$we have that $a \wedge b=0$ if and only if $a \leq b \rightarrow 0$. Moreover, it follows from [1, Theorem 1 , Sec. 3, Ch. VIII] that $\mathrm{DLI}_{\neg}^{+}$is a variety.

Example 3.6. Consider the boolean lattice $B_{2}$, whose atoms are $a$ and $b$. We have that $\left(B_{2}, \rightarrow\right) \in \mathrm{DLI}^{+}$, where $\rightarrow$ is the implication given in Example 3.3. Moreover, $a \wedge b=0$ and $a \rightarrow 0=0$. Thus, $b \not \leq a \rightarrow 0$. Hence, the condition (A) is not satisfied for this algebra. Therefore, $\mathrm{DLI}_{\neg}^{+}$is a proper subvariety of $\mathrm{DLI}^{+}$.

Let $\left(H_{3}, \rightarrow\right)$ be the algebra given in Example 3.4, which is not in $\mathrm{DLI}_{1}^{+}$. Straightforward computations show that $\left(H_{3}, \rightarrow\right) \in \mathrm{DLI}_{\neg}^{+}$.

Finally, consider the bounded distributive lattice $B_{2}$ given in Example 3.6. Define the following binary operation:

\begin{tabular}{c|cccc}
$\rightarrow$ & 0 & $a$ & $b$ & 1 \\
\hline 0 & 1 & 1 & 1 & 1 \\
$a$ & $b$ & 1 & $b$ & 1 \\
$b$ & 0 & 0 & 1 & 1 \\
1 & 0 & 0 & $b$ & 1
\end{tabular}

Then $\left(B_{2}, \rightarrow\right) \in \mathrm{SRL}$ and it is not an algebra of $\mathrm{DLI}_{\neg}^{+}$because $a \wedge b=0$, $b \rightarrow 0=0$ and $a \not \leq 0$.

The relation among all mentioned varieties is depicted in Figure 2. 


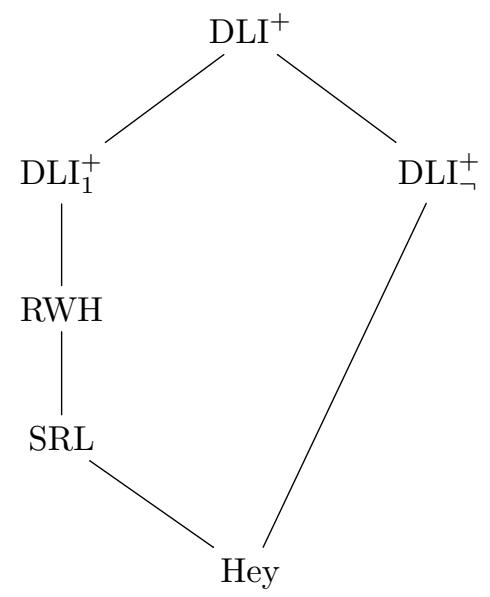

FIGURE 2. The relations among some of the varieties mentioned in this work.

\section{Kalman's functor}

The fact that Kalman's construction can be extended consistently to Heyting algebras led us to believe that some of this picture could be lifted to the variety $\mathrm{DLI}^{+}$. In this section, we study this level of generality.

Let $H \in \mathrm{DLI}^{+}$. Write $\rightarrow$ for the implication of $H$. We define a binary operation on $\mathrm{K}(H)$ (also written $\rightarrow$ ) by

$$
(a, b) \rightarrow(d, e):=((a \rightarrow d) \wedge(e \rightarrow b), a \wedge e) .
$$

This definition is motivated by Remark 2.10. Since $a \wedge b=d \wedge e=0$, then $(a \rightarrow d) \wedge(e \rightarrow b) \wedge a \wedge e=0$. Hence, $\rightarrow$ is well defined in $\mathrm{K}(H)$. Thus, we can consider algebras $(\mathrm{K}(H), \wedge, \vee, \rightarrow, \sim,(0,0),(0,1),(1,0))$ of type $(2,2,2,1,0,0,0)$ where the reduct $(\mathrm{K}(H), \wedge, \vee, \sim,(0,0),(0,1),(1,0))$ is a centered Kleene algebra with the operations defined in Remark 2.1. The next definition is motivated by the original Kalman construction.

Definition 4.1. We write KLI for the category whose objects are algebras $(T, \wedge, \vee, \rightarrow, \sim, \mathrm{c}, 0,1)$ of type $(2,2,2,1,0,0,0)$ such that $(T, \wedge, \vee, \sim, \mathrm{c}, 0,1)$ is a centered Kleene algebra and $\rightarrow$ is a binary operation on $T$ which satisfies the following conditions.

(KLI1) $(T, \wedge, \vee, \rightarrow, 0,1)$ is a DLI-algebra.

$(\mathrm{KLI} 2)(x \wedge(x \rightarrow y)) \vee \mathrm{c} \leq y \vee \mathrm{c}$ for every $x, y$.

(KLI3) $\mathrm{c} \rightarrow \mathrm{c}=1$.

(KLI4) $(x \rightarrow y) \wedge \mathrm{c}=(\sim x \vee y) \wedge \mathrm{c}$ for every $x, y$.

$(\mathrm{KLI} 5)(x \rightarrow \sim y) \vee \mathrm{c}=((x \rightarrow(\sim y \vee \mathrm{c})) \wedge(y \rightarrow(\sim x \vee \mathrm{c}))$, for every $x, y$.

The objects of this category are called Kleene lattices with implication. 
Remark 4.2. Let $H$ be a Kleene algebra endowed with a binary operation $\rightarrow$. We define another binary operation on $H$ by $x * y=\sim(x \rightarrow \sim y)$. Note also that $*$ defines $\rightarrow$. More concretely, $x * y=\sim(x \rightarrow \sim y)$ implies that $x \rightarrow y=\sim(x * \sim y)$.

Let $T$ be a centered Kleene algebra endowed with two binary operations, $\rightarrow$ and $*$, related as in Remark 4.2. Then (KLI1) holds on $T$ if and only if $(T, \wedge, \vee, *, 0,1)$ is a DLF-algebra [6], (KLI2) holds on $T$ if and only if we have $x \wedge \mathrm{c} \leq(\sim y \vee(y * \mathrm{c})) \wedge \mathrm{c}$ for every $x, y,(\mathrm{KLI} 3)$ holds on $T$ if and only $c * \mathrm{c}=0$, (KLI4) holds on $T$ if and only if $(x * y) \vee \mathrm{c}=(x \wedge y) \vee \mathrm{c}$ for every $x, y$, and (KLI5) holds on $T$ if and only if $(x * y) \wedge \mathrm{c}=(x *(y \wedge \mathrm{c})) \vee(y *(x \wedge \mathrm{c}))$ for every $x, y$.

Proposition 4.3. Let $H \in \mathrm{DLI}^{+}$. Then $\mathrm{K}(H)$ admits the structure of a Kleene lattice with the operations defined in Remark 2.1 and the implication given in (4.1). Furthermore, $\mathrm{K}$ extends to a functor from $\mathrm{DLI}^{+}$to $\mathrm{KLI}$, which we also will write $\mathrm{K}$.

Proof. It is a routine exercise to prove the condition (KLI1) (it is an immediate consequence of the fact that $H$ is a $D L I$-algebra).

Let $(a, b),(d, e) \in \mathrm{K}(H)$. In order to prove (KLI2), we use (I5):

$$
\begin{aligned}
& ((a, b) \wedge((a, b) \rightarrow(d, e))) \vee \mathrm{c}=((a, b) \wedge((a \rightarrow d) \wedge(e \rightarrow b), a \wedge e)) \vee \mathrm{c} \\
& \quad=(a \wedge(a \rightarrow d) \wedge(e \rightarrow b), 0) \leq(d \wedge(e \rightarrow b), 0) \leq(d, 0)=(d, e) \wedge \mathrm{c} .
\end{aligned}
$$

Straightforward computations prove (KLI3). In order to prove (KLI4), note that $((a, b) \rightarrow(d, e)) \wedge \mathrm{c}=(0, a \wedge e)$ and

$$
(\sim(a, b) \vee(d, e)) \wedge \mathrm{c}=((b, a) \vee(d, e)) \wedge \mathrm{c}=(0, a \wedge e) .
$$

Hence, we have that $((a, b) \rightarrow(d, e)) \wedge \mathrm{c}=(\sim(a, b) \vee(d, e)) \wedge \mathrm{c}$. Finally, we shall prove (KLI5). We have that

$$
((a, b) \rightarrow \sim(d, e)) \vee \mathrm{c}=((a, b) \rightarrow(e, d)) \vee \mathrm{c}=((a \rightarrow e) \wedge(d \rightarrow b), 0) .
$$

Moreover, we have that

$$
\begin{aligned}
((a, b) \rightarrow(\sim(d, e) \vee c)) \wedge((d, e) \rightarrow(\sim(a, b) \vee c)) & =(a \rightarrow e, 0) \wedge(d \rightarrow b, 0) \\
& =((a \rightarrow e) \wedge(d \rightarrow b), 0)
\end{aligned}
$$

Thus,

$$
((a, b) \rightarrow \sim(d, e)) \vee \mathrm{c}=((a, b) \rightarrow(\sim(d, e) \vee \mathrm{c})) \wedge((d, e) \rightarrow(\sim(a, b) \vee \mathrm{c}))
$$

Then $\mathrm{K}(H)$ is a Kleene lattice.

Let $f: H \rightarrow G$ be a morphism in $\mathrm{DLI}^{+}$. As before, let $\mathrm{K}(f): \mathrm{K}(H) \rightarrow \mathrm{K}(G)$ be defined by $\mathrm{K}(f)(a, b)=(f(a), f(b))$. Straightforward computations show that $K(f)$ preserves the implication operation, which implies that $K(f)$ is a morphism in KLI.

Proposition 4.4. The category of centered Nelson lattices is a full subcategory of KLI. 
Proof. Let $T$ be a centered Nelson lattice. Using Theorem 2.9, it can be proved that $T \cong \mathrm{K}(\mathrm{C}(T))$ in the category of centered Nelson lattices. But $\mathrm{C}(T)$ is a Heyting algebra, so we have $\mathrm{C}(T) \in \mathrm{DLI}^{+}$. Since $\mathrm{K}$ is a functor from $\mathrm{DLI}^{+}$to KLI, we have that $\mathrm{K}(\mathrm{C}(T)) \in \mathrm{KLI}$. Therefore, $T \in \mathrm{KLI}$.

The following lemma will be used throughout the paper.

Lemma 4.5. Let $T \in \mathrm{KLI}$. The binary operation $*$ is monotonic.

Proof. Let $x, y, z \in T$ such that $x \leq y$. Taking into account that $T$ is a $D L I$ algebra, we obtain that $(x \rightarrow \sim z) \wedge(y \rightarrow \sim z)=(x \vee y) \rightarrow \sim z$. But $x \vee y=y$, so $y \rightarrow \sim z \leq x \rightarrow \sim z$, i.e., $\sim(y * z) \leq \sim(x * z)$. This implies that $x * z \leq y * z$, which was our aim.

In what follows, we give some algebraic properties of the variety KLI which we shall use later.

Lemma 4.6. Let $T \in \mathrm{KLI}$.

(1) For every $x, \mathrm{c} * x \leq \mathrm{c}$. In particular, $\mathrm{c} \leq \mathrm{c} \rightarrow x$ for every $x$.

(2) For every $x, x * y=y * x$.

(3) For every $x, x *(x \rightarrow \mathrm{c}) \leq \mathrm{c}$. In particular, for every $x, y$, if $x \leq y \rightarrow \mathrm{c}$, then $x * y \leq \mathrm{c}$.

(4) For every $x,(\mathrm{c} \rightarrow x) * \sim x \leq \mathrm{c}$.

(5) For every $x, \mathrm{c} \rightarrow(\mathrm{c} \vee x)=1$.

(6) For every $x, x \rightarrow y=\sim y \rightarrow \sim x$.

(7) $\mathrm{c} \rightarrow 0=\mathrm{c}$. Equivalently, $\mathrm{c} * 1=c$.

Proof. (1): Let $x \in T$. Then $\mathrm{c} * x \leq \mathrm{c} * 1=\mathrm{c}$, so $\mathrm{c} * x \leq \mathrm{c}$. In order to prove that $\mathrm{c} \leq \mathrm{c} \rightarrow x$, note that $\mathrm{c} * \sim x \leq \mathrm{c}$. So, $\mathrm{c} \leq \mathrm{c} \rightarrow x$.

(2): Let $x, y \in T$. From (KLI4) and (KLI5), we have $(x * y) \wedge \mathrm{c}=(y * x) \wedge \mathrm{c}$ and $(x * y) \vee \mathrm{c}=(y * x) \vee \mathrm{c}$. So, we have that $x * y=y * x$.

(3): Let $x, y \in T$. It follows from (KLI4) that we have

$$
(x *(x \rightarrow \mathrm{c})) \vee \mathrm{c}=(x \wedge(x \rightarrow \mathrm{c})) \vee \mathrm{c}=\mathrm{c},
$$

so $x *(x \rightarrow \mathrm{c}) \leq \mathrm{c}$. Now suppose that $x \leq y \rightarrow \mathrm{c}$. Then $x * y \leq y *(y \rightarrow \mathrm{c}) \leq \mathrm{c}$, i.e., $x * y \leq$ c.

(4): Let $x \in T$. Since $\mathrm{c} \rightarrow x=\sim x \rightarrow \mathrm{c}$, by (3) we have $(\mathrm{c} \rightarrow x) * \sim x \leq \mathrm{c}$.

(5): Let $x \in T$. We have that $\mathrm{c} \wedge \sim x \leq \mathrm{c}$, so $\mathrm{c} *(\mathrm{c} \wedge \sim x) \leq \mathrm{c}^{2}=0$. Hence, we have that $\mathrm{c} *(\mathrm{c} \wedge \sim x)=0$, i.e., $\mathrm{c} \rightarrow(\mathrm{c} \vee x)=1$.

(6): Let $x, y \in T$. By (KLI4) and (KLI5), $(x \rightarrow y) \wedge \mathrm{c}=(\sim y \rightarrow \sim x) \wedge \mathrm{c}$ and $(x \rightarrow y) \vee \mathrm{c}=(\sim y \rightarrow \sim x) \vee \mathrm{c}$, so $x \rightarrow y=\sim y \rightarrow \sim x$.

(7): We shall prove that $\mathrm{c} \rightarrow 0=0$, which is equivalent to $1 \rightarrow \mathrm{c}=\mathrm{c}$. It follows from $(\mathrm{KLI} 2)$ that $(1 \wedge(1 \rightarrow \mathrm{c})) \vee \mathrm{c} \leq \mathrm{c} \vee \mathrm{c}$, so $(1 \rightarrow \mathrm{c}) \vee \mathrm{c}=\mathrm{c}$. Then $1 \rightarrow \mathrm{c} \leq \mathrm{c}$. Also, it follows from (KLI4) that $(1 \rightarrow \mathrm{c}) \wedge \mathrm{c}=(\sim 1 \vee \mathrm{c}) \wedge \mathrm{c}$, i.e., $(1 \rightarrow \mathrm{c}) \wedge \mathrm{c}=\mathrm{c}$. Then $\mathrm{c} \leq 1 \rightarrow \mathrm{c}$. Therefore, $1 \rightarrow \mathrm{c}=\mathrm{c}$.

By Lemma 4.6 we can prove the following 
Proposition 4.7. If $(T, \vee, \wedge, \sim, \rightarrow, \mathrm{c}, 0,1) \in \mathrm{KLI}$, then $(\mathrm{C}(T), \vee, \wedge, \rightarrow, \mathrm{c}, 1) \in$ $\mathrm{DLI}^{+}$. If $f: T \rightarrow U$ is a morphism in $\mathrm{KLI}$, then $\mathrm{C}(f): \mathrm{C}(T) \rightarrow \mathrm{C}(U)$, given by $\mathrm{C}(f)(x)=f(x)$, is a morphism in $\mathrm{DLI}^{+}$.

Proof. We have that $\mathrm{C}(T)$ is closed under the operation $\rightarrow$. In order to prove it, let $x, y \geq \mathrm{c}$. Then $x * \sim y \leq x * \mathrm{c}=\mathrm{c} * x \leq \mathrm{c}$, so $x \rightarrow y=\sim(x * \sim y) \geq \mathrm{c}$. Let $x \geq \mathrm{c}$. Then $\sim x \leq \mathrm{c}$, so $\mathrm{c} * \sim x \leq \mathrm{c}^{2}=0$. Thus, we have that $c \rightarrow x=1$. Finally, let $x, y \geq \mathrm{c}$. Since $x \wedge(x \rightarrow y)=(x \wedge(x \rightarrow y)) \vee \mathrm{c} \leq y \vee \mathrm{c}=y$, then $x \wedge(x \rightarrow y) \leq y$. The rest of the proof is immediate.

It can be seen that $\mathrm{C}$ defines a functor from KLI to $\mathrm{DLI}^{+}$.

Remark 4.8. For $H \in \mathrm{DLI}^{+}$and $a, b \in H, \alpha_{H}(a \rightarrow b)=\alpha_{H}(a) \rightarrow \alpha_{H}(b)$, where $\alpha_{H}$ is the map defined in Remark 2.2. Then $\alpha_{H}$ is an isomorphism in $\mathrm{DLI}^{+}$.

For the case of the category KLI, we can show the following

Proposition 4.9. Let $T \in \mathrm{KLI}$. Then $\beta_{T}$ is a morphism in KLI, where $\beta_{T}$ is the map defined in Remark 2.2.

Proof. We need to prove that $\beta_{T}(x \rightarrow y)=\beta_{T}(x) \rightarrow \beta_{T}(y)$ for every $x, y$. Equivalently, we need to prove that $\beta_{T}(x \rightarrow \sim y)=\beta_{T}(x) \rightarrow \beta_{T}(\sim y)$ for every $x, y$. First note that

$$
\begin{aligned}
\beta_{T}(x \rightarrow \sim y) & =((x \rightarrow \sim y) \vee \mathrm{c}, \sim(x \rightarrow \sim y) \vee \mathrm{c}) \\
& =(\sim(x * y) \vee \mathrm{c},(x * y) \vee \mathrm{c})=(\sim((x * y) \wedge \mathrm{c}),(x * y) \vee \mathrm{c}) .
\end{aligned}
$$

It follows from Lemma 4.6 that $\mathrm{c} \rightarrow(\sim x \vee \mathrm{c})=1$ and $\mathrm{c} \rightarrow(\sim y \vee \mathrm{c})=1$. Then we also have that

$$
\begin{aligned}
\beta_{T}(x) \rightarrow \beta_{T}(\sim y) & =((x \rightarrow(\sim y \vee c) \wedge(y \rightarrow(\sim x \vee c)),(x \wedge y) \vee c) \\
& =(\sim((x *(y \wedge \mathrm{c})) \wedge(y *(x \wedge \mathrm{c})),(x \wedge y) \vee \mathrm{c}) .
\end{aligned}
$$

Thus, from (KLI4) and (KLI5), we have $\beta_{T}(x \rightarrow \sim y)=\beta_{T}(x) \rightarrow \beta_{T}(\sim y)$.

For every $(T, \wedge, \vee, \sim, \mathrm{c}, 0,1) \in \mathrm{Kl}_{\mathrm{c}}$, it is possible to define a binary operation $\rightarrow$ such that $(T, \wedge, \vee, \rightarrow, \sim, \mathrm{c}, 0,1) \in \mathrm{KLI}$. We do this in the following example.

Example 4.10. Let $(T, \wedge, \vee, \sim, \mathrm{c}, 0,1) \in \mathrm{Kl}_{\mathrm{c}}$. Define on $T$ the following binary map:

$$
x \rightarrow y= \begin{cases}1, & \text { if } x \leq \mathrm{c} \text { and } y \geq \mathrm{c} ; \\ \sim x, & \text { if } x \leq \mathrm{c} \text { and } y \geq \mathrm{c} ; \\ y, & \text { if } x \not \leq \mathrm{c} \text { and } y \geq \mathrm{c} ; \\ ((y \vee \mathrm{c}) \wedge \sim x) \vee((\sim x \vee \mathrm{c}) \wedge y), & \text { if } x \not \leq \mathrm{c} \text { and } y \geq \mathrm{c} .\end{cases}
$$

It can be seen that $(T, \wedge, \vee, \rightarrow, \sim, \mathrm{c}, 0,1) \in \mathrm{KLI}$.

Remark 4.11. Note that for the centered Kleene algebra of the Example 2.5, Example 4.10 yields an algebra of KLI which does not satisfy the condition (CK). 
We write $\mathrm{KLI}^{\prime}$ for the full subcategory of KLI whose objects satisfy the condition $(\mathrm{CK})$.

Theorem 4.12. There exists a categorical equivalence $\mathrm{K} \dashv \mathrm{C}: \mathrm{DLI}^{+} \rightarrow \mathrm{KLI}^{\prime}$. Proof. This follows from Theorem 2.7, Proposition 4.3, Proposition 4.7, Propo sition 4.9, and Remark 4.8.

Remark 4.13. For algebras of $\mathrm{KLI}^{\prime}$, the involution can not necessarily be defined in terms of the implication and some constant. Consider the boolean algebra of four elements $\{0, a, b, 1\}$, where $a$ and $b$ are the atoms. For the underlying bounded lattice, consider the implication given in Example 3.3, and write $B_{4}$ for this algebra. We have that $\mathrm{K}\left(B_{4}\right) \in \mathrm{KLI}^{\prime}$. For $x=(a, b)$, the only $y \in \mathrm{K}(L)$ such that $x \rightarrow y=\sim x$ is $y=\sim x$. However, $c \rightarrow \sim c \neq \sim c$. Therefore, there is no $y \in \mathrm{K}(L)$ such that $x \rightarrow y=\sim x$ for every $x \in \mathrm{K}(L)$.

In the rest of this section, we shall study the restriction to the equivalence given in Theorem 4.12 to subvarieties of $\mathrm{DLI}^{+}$defined at the end of Section 3 .

4.1. The variety $\mathrm{DLI}_{1}^{+}$. Let $T \in \mathrm{KLI}^{\prime}$. We also write (I6) for the equation $x \rightarrow x=1$ on $T$. Let $H \in \mathrm{DLI}^{+}$and $T \in \mathrm{KLI}^{\prime}$. If $H$ satisfies (I6), then $\mathrm{K}(H)$ satisfies (I6), and if $T$ satisfies (I6), then $\mathrm{C}(T)$ satisfies (I6).

Corollary 4.14. There exists a categorical equivalence between $\mathrm{DLI}_{1}^{+}$and the full subcategory of $\mathrm{KLI}^{\prime}$ whose objects satisfy (I6).

Proof. This follows from Theorem 4.12.

4.2. The variety RWH. For $T \in \mathrm{KLI}$, we consider the following equation: $(\mathrm{KLI} 7)(x \rightarrow y) \wedge(y \rightarrow z) \leq(x \rightarrow z) \vee \mathrm{c}$.

Lemma 4.15. Let $H \in \mathrm{DLI}^{+}$and $T \in \mathrm{KLI}$. If $H$ satisfies (I7), then $\mathrm{K}(H)$ satisfies (KLI7), and if $T$ satisfies (KLI7), then $\mathrm{C}(T)$ satisfies (I7).

Proof. First we suppose that $H$ satisfies (I7). Let $(a, b),(d, e),(f, g) \in \mathrm{K}(H)$. We have that

$$
\begin{aligned}
& ((a, b) \rightarrow(d, e)) \wedge((d, e) \rightarrow(f, g)) \\
\leq & ((a \rightarrow d) \wedge(d \rightarrow f) \wedge(g \rightarrow e) \wedge(e \rightarrow b), 0) \\
\leq & ((a \rightarrow f) \wedge(g \rightarrow b), 0)=((a, b) \rightarrow(f, g)) \vee c .
\end{aligned}
$$

Conversely, assume that $T$ satisfies (KLI7). Let $x, y, z \geq \mathrm{c}$. Then

$$
(x \rightarrow y) \wedge(y \rightarrow z) \leq x \rightarrow z=(x \rightarrow z) \vee \mathrm{c} .
$$

Corollary 4.16. There exists a categorical equivalence between RWH and the full subcategory of $\mathrm{KLI}^{\prime}$ whose objects satisfy (I6) and (KLI7).

Proof. This follows from Lemma 4.15 and Theorem 4.12. 
4.3. The variety SRL. For $T \in$ KLI we consider the following equation:

$(\mathrm{KLI} 8) x \rightarrow y \leq(z \vee \mathrm{c}) \rightarrow(x \rightarrow y)$.

Lemma 4.17. Let $H \in \mathrm{DLI}^{+}$and $T \in \mathrm{KLI}$. If $H$ satisfies (I8) then $\mathrm{K}(H)$ satisfies (KLI8), and if $T$ satisfies (KLI8) then $\mathrm{C}(T)$ satisfies (I8).

Proof. First suppose that $H$ satisfies (I8). Let $(a, b),(d, e),(f, g) \in \mathrm{K}(H)$. We have that

$$
\begin{aligned}
(a, b) \rightarrow(d, e) & =((a \rightarrow d) \wedge(e \rightarrow b), a \wedge e) \\
\leq & (f \rightarrow((a \rightarrow d) \wedge(e \rightarrow b)), a \wedge e) .
\end{aligned}
$$

The implication $(f, 0) \rightarrow((a \rightarrow d) \wedge(e \rightarrow b), a \wedge e)$ yields

$$
(f \rightarrow((a \rightarrow d) \wedge(e \rightarrow b)) \wedge((a \wedge e) \rightarrow 0), f \wedge a \wedge e) .
$$

Then one has, as desired,

$$
\begin{aligned}
(f \rightarrow((a \rightarrow d) \wedge(e \rightarrow b)) \wedge((a \wedge e) & \rightarrow 0), f \wedge a \wedge e) \\
& \leq(f \rightarrow((a \rightarrow d) \wedge(e \rightarrow b)), f \wedge a \wedge e) .
\end{aligned}
$$

Conversely, let $T$ satisfies (KLI8). Let $x, y, z \geq \mathrm{c}$. Then $z \vee \mathrm{c}=z$. Hence,

$$
x \rightarrow y \leq(z \vee c) \rightarrow(x \rightarrow y)=z \rightarrow(x \rightarrow y) .
$$

Corollary 4.18. There exists a categorical equivalence between SRL and the full subcategory of $\mathrm{KLI}^{\prime}$ whose objects satisfy (I6), (KLI7) and (KLI8).

Proof. This follows from Lemma 4.17 and Corollary 4.16.

4.4. The variety $\mathrm{DLI}_{\neg}^{+}$. Let $T \in \mathrm{KLI}$. We consider the following condition:

(B) For every $x, y$, if $x, y \geq \mathrm{c}$ and $x \wedge y \leq \mathrm{c}$, then $y \leq x \rightarrow \mathrm{c}$.

Lemma 4.19. If $H \in \mathrm{DLI}^{+}$satisfies (A), then $\mathrm{K}(H)$ satisfies (B). Conversely, if $T \in \mathrm{KLI}$ satisfies $(\mathrm{B})$, then $\mathrm{C}(T)$ satisfies $(\mathrm{A})$.

Proof. Let $H \in \mathrm{DLI}^{+}$satisfy (A). Let $x=(a, d), y=(b, e) \in \mathrm{K}(H)$ such that $x, y \geq \mathrm{c}$ and $x \wedge y \leq \mathrm{c}$. Hence, $x=(a, 0), y=(b, 0)$ and $a \wedge b=0$. Thus, it follows from (A) that $a \leq b \rightarrow 0$. But we have that $y \rightarrow c=(b \rightarrow 0,0)$, so $x \leq y \rightarrow$ c. Conversely, suppose that $T \in$ KLI satisfies (B). Let $x, y \in \mathrm{C}(T)$ such that $x \wedge y=\mathrm{c}$. It follows from (B) that $y \leq x \rightarrow \mathrm{c}$. Therefore, we obtain the condition $(\mathrm{A})$ in $\mathrm{C}(T)$.

Lemma 4.20. Let $T \in \mathrm{KLI}$. If $T$ satisfies (B), then $T$ satisfies (CK).

Proof. Let $x, y \geq \mathrm{c}$ such that $x \wedge y \leq \mathrm{c}$. It follows from (B) that $x \leq y \rightarrow \mathrm{c}$. Let $z=x \wedge(y \rightarrow \sim y)$. Then it follows from (KLI4) that

$$
\mathrm{c} \wedge z=\mathrm{c} \wedge x \wedge(y \rightarrow \sim y)=\mathrm{c} \wedge(y \rightarrow \sim y)=\mathrm{c} \wedge \sim y=\sim y .
$$


Then we have that $\sim z \vee c=y$. From (KLI5) and Lemma 4.6, we have

$$
\begin{aligned}
z \vee \mathrm{c} & =(x \wedge(y \rightarrow \sim y)) \vee \mathrm{c}=(x \wedge(y \rightarrow \sim y)) \vee(\mathrm{c} \wedge x) \\
& =x \wedge((y \rightarrow \sim y) \vee \mathrm{c})=x \wedge(y \rightarrow(\sim y \vee \mathrm{c})) \wedge(y \rightarrow(\sim y \vee \mathrm{c})) \\
& =x \wedge(y \rightarrow(\sim y \vee \mathrm{c}))=x \wedge(y \rightarrow \mathrm{c})=x .
\end{aligned}
$$

Thus, we obtain that $z \vee \mathrm{c}=x$. Therefore, $T$ satisfies $(\mathrm{CK})$.

The next theorem follows from Theorem 4.12, Lemma 4.19 and Lemma 4.20.

Theorem 4.21. There exists an equivalence between $\mathrm{DLI}_{\neg}^{+}$and the full subcategory of KLI whose objects satisfies (B).

Let $T \in$ KLI. Does (CK) imply (B)? Suppose that (CK) implies (B). By previous results of this paper, we obtain $\mathrm{DLI}^{+}=\mathrm{DLI}_{\neg}^{+}$, which is a contradiction. Therefore, (CK) does not necessarily imply (B).

4.5. Conclusions. Let $L \in \mathrm{DLI}^{+}$. Then $\mathrm{K}(L) \in \mathrm{Kl}_{\mathrm{c}}^{\prime}$. In what follows, we summarize in a table part of the results of this section.

\begin{tabular}{c|c} 
Condition in $L$ & Condition in $\mathrm{K}(L)$ \\
\hline (I6) & $(\mathrm{I} 6)$ \\
$(\mathrm{I} 7)$ & $(\mathrm{KLI} 7)$ \\
$(\mathrm{I} 8)$ & $(\mathrm{KLI} 8)$ \\
(A) & $(\mathrm{B})$
\end{tabular}

The condition on the left holds in $L$ if and only if the condition on the right holds in $\mathrm{K}(L)$.

\section{Congruences}

Write $\operatorname{Con}(H)$ for the lattice of congruences of an algebra $H$. It was proved in [4] that if $H$ is a bounded distributive lattice, then there exists an order isomorphism between $\operatorname{Con}(H)$ and $\operatorname{Con}(\mathrm{K}(H))$. In this section, we make explicit this construction in order to extend this property to the case of $\mathrm{DLI}^{+}$.

Let $H$ be a $D L I$-algebra. We say that $F$ is a filter of $H$ if $F$ is a lattice filter of $H$, meaning that $1 \in F, x \wedge y \in F$ whenever $x, y \in F$, and if $x \leq y$ and $x \in F$, then $y \in F$. It is known that in any Heyting algebra $H$, there exists an order isomorphism between $\operatorname{Con}(H)$ and the filters of $H$ [1]. Moreover, it was proved in [7, Proposition 6.12] that for $H \in \mathrm{RWH}$, there exists an order isomorphism between $\operatorname{Con}(H)$ and the filters $F$ of $H$ such that $1 \rightarrow f \in F$ whenever $f \in F$. In this section, we also generalize these properties proving that there exists an order isomorphism between $\operatorname{Con}(H)$ and certain family of filters of $H$, whenever $H \in \mathrm{DLI}_{1}^{+}$.

We start with some preliminary definitions. 
Definition 5.1. Let $H$ be a bounded distributive lattice and $\theta$ a congruence of $H$. We define in $\mathrm{K}(H)$ the following congruence:

$$
((a, b),(d, e)) \in \tau_{\theta} \text { if and only if }(a, d) \in \theta \text { and }(b, e) \in \theta .
$$

If $\tau$ is a congruence in $\mathrm{K}(H)$, we define in $H$ the following congruence:

$$
(a, b) \in \theta^{\tau} \text { if and only if }((a, 0),(b, 0)) \in \tau .
$$

Then we have the following lemma, which is inspired by [4, Remark 7.12].

Lemma 5.2. For a bounded distributive lattice $H, f: \operatorname{Con}(H) \rightarrow \operatorname{Con}(\mathrm{K}(H))$, given by $f(\theta)=\tau_{\theta}$, is an order isomorphism.

Proof. Let $\theta_{1}, \theta_{2} \in \operatorname{Con}(H)$ such that $\tau_{\theta_{1}}=\tau_{\theta_{2}}$, and let $(a, b) \in \theta_{1}$. Since $((a, 0),(b, 0)) \in \tau_{\theta_{1}}$, then $(a, b) \in \theta_{2}$. Thus, $f$ is an injective map.

Let $\tau \in \operatorname{Con}(\mathrm{K}(H))$. We prove that $f\left(\theta^{\tau}\right)=\tau$. Let $((a, b),(d, e)) \in f\left(\theta^{\tau}\right)$, i.e., $(a, d) \in \theta^{\tau}$ and $(b, e) \in \theta^{\tau}$, which means that $((a, 0),(d, 0)) \in \tau$ and $((b, 0),(e, 0)) \in \tau$. Since $((a, b) \wedge \mathrm{c},(d, e) \wedge \mathrm{c}),((a, b) \vee \mathrm{c},(d, e) \vee \mathrm{c}) \in \tau$, then $((a, b),(d, e)) \in \tau$. Thus, $f\left(\theta^{\tau}\right) \subseteq \tau$. In order to prove the converse inclusion, let $((a, b),(d, e)) \in \tau$. We need to show that $(a, d) \in \theta^{\tau}$ and $(b, e) \in \theta^{\tau}$, which means that $((a, 0),(d, 0)) \in \tau$ and $((b, 0),(e, 0)) \in \tau$. As $((a, b),(d, e)) \in \tau$, we obtain that $((a, b) \vee \mathrm{c},(d, e) \vee c) \in \tau$ and $((a, b) \wedge \mathrm{c},(d, e) \wedge c) \in \tau$, i.e., $((a, 0),(d, 0)) \in \tau$ and $((0, b),(0, e)) \in \tau$. In particular, $(\sim(0, b), \sim(0, e)) \in \tau$, i.e., $((b, 0),(e, 0)) \in \tau$. Then $\tau \subseteq f\left(\theta^{\tau}\right)$. Hence, $f\left(\theta^{\tau}\right)=\tau$, which implies that $f$ is a surjective map.

Finally, taking into account that $\theta_{1} \subseteq \theta_{2}$ if and only if $f\left(\theta_{1}\right) \subseteq f\left(\theta_{2}\right)$, we conclude that $f$ is an order isomorphism.

Lemma 5.3. Let $H \in \mathrm{DLI}^{+}, \theta \in \operatorname{Con}(H)$, and $\tau \in \operatorname{Con}(\mathrm{K}(H))$. Then $\tau_{\theta} \in \operatorname{Con}(\mathrm{K}(H))$ and $\theta^{\tau} \in \operatorname{Con}(H)$.

Proof. Let $\left(\left(a_{1}, b_{1}\right),\left(d_{1}, e_{1}\right)\right) \in \tau_{\theta},\left(\left(a_{2}, b_{2}\right),\left(d_{2}, e_{2}\right)\right) \in \tau_{\theta}$, i.e., $\left(a_{1}, d_{1}\right) \in \theta$, $\left(a_{2}, d_{2}\right) \in \theta,\left(b_{1}, e_{1}\right) \in \theta$ and $\left(b_{2}, e_{2}\right) \in \theta$. Hence, $\left(a_{1} \rightarrow a_{2}, d_{1} \rightarrow d_{2}\right) \in \theta$, $\left(b_{2} \rightarrow b_{1}, e_{2} \rightarrow e_{1}\right) \in \theta$ and $\left(a_{1} \wedge b_{2}, d_{1} \wedge e_{2}\right) \in \theta$. Moreover, we have that $\left(\left(a_{1} \rightarrow a_{2}\right) \wedge\left(b_{2} \rightarrow b_{1}\right),\left(d_{1} \rightarrow d_{2}\right) \wedge\left(e_{2} \rightarrow e_{1}\right)\right) \in \theta$. Since $\left(a_{1} \wedge b_{2}, d_{1} \wedge e_{2}\right) \in \theta$, from the definition of $\tau_{\theta}$ we have $\left(\left(a_{1}, b_{1}\right) \rightarrow\left(a_{2}, b_{2}\right),\left(d_{1}, e_{1}\right) \rightarrow\left(d_{2}, e_{2}\right)\right) \in \tau_{\theta}$. Then, $\tau_{\theta} \in \operatorname{Con}(\mathrm{K}(H))$.

Finally let $\tau \in \operatorname{Con}(\mathrm{K}(H))$. Let $(a, b) \in \theta^{\tau}$ and $(d, e) \in \theta^{\tau}$. Hence, $((a, 0),(b, 0)),((d, 0),(e, 0)) \in \tau$. Thus, $((a, 0) \rightarrow(d, 0),(b, 0) \rightarrow(e, 0)) \in \tau$. But $(a, 0) \rightarrow(d, 0)=(a \rightarrow d, 0)$ and $(b, 0) \rightarrow(e, 0)=(b \rightarrow e, 0)$, so we have that $(a \rightarrow d, b \rightarrow e) \in \theta^{\tau}$. Therefore, $\theta^{\tau} \in \operatorname{Con}(H)$.

Corollary 5.4. Let $H \in \mathrm{DLI}^{+}$. The map $f: \operatorname{Con}(H) \rightarrow \operatorname{Con}(\mathrm{K}(H))$, given by $f(\theta)=\tau_{\theta}$, is an order isomorphism.

As we have said before, congruences in $\mathrm{DLI}_{1}^{+}$are in correspondence with certain family of filters. In what follows, we are going to make this statement precise. 
Definition 5.5. Let $H \in \mathrm{DLI}_{1}^{+}$and $F$ a filter. We say that $F$ is an open filter if $1 \rightarrow f \in F$ whenever $f \in F$.

The following family of filters will play an important role.

Definition 5.6. Let $H \in \mathrm{DLI}_{1}^{+}$and $F$ a filter. We say that $F$ is a 1-filter if $((a \wedge f) \rightarrow b) \rightarrow(a \rightarrow b) \in F$ whenever $a, b \in H$ and $f \in F$.

Let $H \in \mathrm{DLI}_{1}^{+}$. For $\theta \in \operatorname{Con}(H)$ and $F$ a filter of $H$, we define

$$
\Theta(F)=\{(a, b) \in H \times H: a \wedge f=b \wedge f \text { for some } f \in F\} .
$$

If $a \in H$ and $\theta \in \operatorname{Con}(H)$, we write $a / \theta$ for the equivalence class of $a$ associated to $\theta$. Straightforward computations show that $1 / \theta$ is a filter, $\theta=\Theta(1 / \theta)$, $F=1 / \Theta(F)$, and $\Theta(F)$ is a congruence with respect to the lattice operations. Moreover, if $F$ and $G$ are filters, then $F \subseteq G$ if and only if $\Theta(F) \subseteq \Theta(G)$.

Proposition 5.7. Let $H \in \mathrm{DLI}_{1}^{+}$. The correspondence $F \mapsto \Theta(F)$ defines an order isomorphism between the lattice of 1-filters of $H$ and $\operatorname{Con}(H)$.

Proof. Let $\theta \in \operatorname{Con}(H)$. We shall prove that for every $a, b \in H$ and $f \in 1 / \theta$, $((a \wedge f) \rightarrow b) \rightarrow(a \rightarrow b) \in 1 / \theta$. Let $f \in 1 / \theta$, i.e., $(f, 1) \in \theta$. Then $(a \wedge f, a) \in \theta$. Hence, $((a \wedge f) \rightarrow b, a \rightarrow b) \in \theta$. But $(a \rightarrow b) \rightarrow(a \rightarrow b)=1$, so we have that $(((a \wedge f) \rightarrow b) \rightarrow(a \rightarrow b), 1) \in \theta$, i.e., $((a \wedge f) \rightarrow b) \rightarrow(a \rightarrow b) \in 1 / \theta$.

Let $F$ be a 1-filter, and let $(a, b),(d, e) \in \Theta(F)$. We only need to prove that $(a \rightarrow d, b \rightarrow e) \in \Theta(F)$. Since $(a, b),(d, e) \in \Theta(F)$, then there exist $f_{1}, f_{2} \in F$ such that $a \wedge f_{1}=b \wedge f_{1}$ and $d \wedge f_{2}=e \wedge f_{2}$. Let $f=f_{1} \wedge f_{2} \in F$, so $a \wedge f=b \wedge f$ and $d \wedge f=e \wedge f$. Let $g=(((a \wedge f) \rightarrow e) \rightarrow(b \rightarrow e))$. Our aim is to prove that $(a \rightarrow d) \wedge g=(b \rightarrow e) \wedge g$. First note that $(a \wedge f) \rightarrow(e \wedge f)=$ $((a \wedge f) \rightarrow e) \wedge((a \wedge f) \rightarrow f)$. But $(a \wedge f) \rightarrow f=1$ because $a \wedge f \leq f$ implies that $(a \wedge f) \rightarrow f \geq f \rightarrow f=1$. Then $(a \wedge f) \rightarrow(e \wedge f)=(a \wedge f) \rightarrow e$. Thus,

$$
g=((a \wedge f) \rightarrow(e \wedge f)) \rightarrow(b \rightarrow e) .
$$

Moreover, we have that

$$
a \rightarrow d \leq(a \wedge f) \rightarrow d
$$

because $a \wedge f \leq a$. Taking into account equations (5.1), (5.2), the equality $(a \wedge f) \rightarrow(d \wedge f)=(a \wedge f) \rightarrow d$ and the inequality $h \wedge(h \rightarrow i) \leq i$ we have

$$
\begin{aligned}
(a \rightarrow d) \wedge g & \leq((a \wedge f) \rightarrow d) \wedge(((a \wedge f) \rightarrow(e \wedge f)) \rightarrow(b \rightarrow e)) \\
& =((a \wedge f) \rightarrow d) \wedge(((a \wedge f) \rightarrow(d \wedge f)) \rightarrow(b \rightarrow e)) \\
& =((a \wedge f) \rightarrow d) \wedge(((a \wedge f) \rightarrow d) \rightarrow(b \rightarrow e)) \leq b \rightarrow e .
\end{aligned}
$$

Hence, $(a \rightarrow d) \wedge g \leq(b \rightarrow e) \wedge g$. Similarly, $(b \rightarrow e) \wedge g \leq(a \rightarrow d) \wedge g$. Thus, we obtain $(a \rightarrow d) \wedge g=(b \rightarrow e) \wedge g$. Therefore, $(a \rightarrow d, b \rightarrow e) \in \Theta(F)$, which was our aim.

Corollary 5.8. Let $H \in \mathrm{DLI}_{1}^{+}$. The map $\tau \mapsto 1 / \theta^{\tau}$ from $\operatorname{Con}(\mathrm{K}(H))$ to the set of 1-filters of $H$ is an order isomorphism.

Proof. This follows from Corollary 5.4 and Proposition 5.7. 
Remark 5.9. Let $H \in \mathrm{DLI}_{1}^{+}$. Then any 1-filter is an open filter since we have the inequality $((1 \wedge f) \rightarrow f) \rightarrow(1 \rightarrow f)=1 \rightarrow(1 \rightarrow f) \leq 1 \rightarrow f$.

Lemma 5.10. Let $H \in \mathrm{DLI}_{1}^{+}, F$ a 1-filter of $H$, and $a, b \in H$. The following facts are equivalent:

(1) $a \wedge f=b \wedge f$ for some $f \in F$;

(2) $a \rightarrow b \in F$ and $b \rightarrow a \in F$.

Proof. (1) $\Rightarrow(2)$ : Suppose that $a \rightarrow b \in F$ and $b \rightarrow a \in F$. Then we have that the element $f=(a \rightarrow b) \wedge(b \rightarrow a) \in F$. Taking into account that $a \wedge(a \rightarrow b) \leq b$, we obtain that $a \wedge f \leq b$, so $a \wedge f \leq b \wedge f$. In a similar way, we have that $b \wedge f \leq a \wedge f$. Then $f \wedge a=f \wedge b$.

$(2) \Rightarrow(1)$ : Suppose that there exists $f \in F$ such that $a \wedge f=b \wedge f$. Taking into account that $a \wedge f \leq b$, we obtain $a \rightarrow(a \wedge f) \leq a \rightarrow b$. Moreover, we have that $a \rightarrow(a \wedge f)=a \rightarrow f$, so $a \rightarrow f \leq a \rightarrow b$. Since $a \leq 1,1 \rightarrow f \leq a \rightarrow f$. Thus, $1 \rightarrow f \leq a \rightarrow b$. Since $F$ is an open filter (Remark 5.9), we have that $a \rightarrow b \in F$. Analogously, we get $b \rightarrow a \in F$.

Corollary 5.11. Let $H \in \mathrm{RWH}$ and $F \subseteq H$. Then $F$ is a 1-filter if and only if $F$ is an open filter.

Proof. This is a straightforward consequence of Proposition 5.7, Remark 5.9, Lemma 5.10, and that $F \mapsto\{(a, b) \in H \times H: a \rightarrow b \in F$ and $b \rightarrow a \in F\}$ defines an order isomorphism between the lattice of open filters of $H$ and $\operatorname{Con}(H)[7$, Proposition 6.12].

Let $H \in \mathrm{DLI}_{1}^{+}$. The question naturally arises whether there is a bijection between $\operatorname{Con}(H)$ and the set of open filters of $H$. In general, the answer is negative. To show this, consider the algebra $H_{4} \in \mathrm{DLI}_{1}^{+}$given in Example 3.5. We have exactly three open filters: $H_{4},\{b, 1\}$, and $\{1\}$. However, we have that $1 \rightarrow b=b \in\{b, 1\}$ and $((1 \rightarrow b) \rightarrow a) \rightarrow(1 \rightarrow a)=a \rightarrow 0=0 \notin\{b, 1\}$. Hence, the open filter $\{b, 1\}$ is not a 1-filter.

\section{Appendix}

As we said before, in [8, Theorem 2.4] it was proved that there exists a categorical equivalence between BDL and the full subcategory of $\mathrm{Kl}_{\mathrm{c}}$ whose objects satisfies the interpolation property. We end this paper by giving a proof of the fact that if $T \in \mathrm{Kl}_{\mathrm{c}}$, then $T$ satisfies the interpolation property [8] if and only if $T$ satisfies (CK) (see Remark 2.8). We start with some preliminary definitions.

A Kleene space [8] is a pair $(X, g)$, with $X$ a Priestley space and $g: X \rightarrow X$ an involutorial homeomorphism which is also an order isomorphism from $X$ onto its order dual. Define $X^{+}=\{x \in X: x \leq g(x)\}$. We say that $(X, g)$ satisfies the interpolation property if given $x, y \in X^{+}$such that $x \leq g(y)$, there exists $z \in X$ such that $x \leq z \leq g(x)$ and $y \leq z \leq g(y)$. If $T$ is a Kleene 
algebra, we write $\mathrm{X}(T)$ for the Priestley space associated to the underlying bounded lattice of $T$. A Kleene algebra $T$ is said to satisfy the interpolation property if the Kleene space $S(T)$ satisfies it, where $S(T)=(\mathrm{X}(T), g)$ and $g: \mathrm{X}(T) \rightarrow \mathrm{X}(T)$ is given by $g(P)=\mathrm{X}(T)-\{\sim a: a \in P\}$. See [8] for details about this construction.

Proposition 6.1. Let $T \in \mathrm{Kl}_{\mathrm{c}}$. Then $T$ satisfies the interpolation property if and only if $T$ satisfies $(\mathrm{CK})$. Moreover, $\mathrm{Kl}_{\mathrm{c}}^{\prime}$ is the full subcategory of $\mathrm{Kl}_{\mathrm{c}}$ whose objects satisfy the interpolation property.

Proof. In this proof, we shall use the following fact for $T, U$ centered Kleene algebras: if $f: T \rightarrow U$ is an isomorphism in the category of Kleene algebras, then $f$ is an isomorphism in $\mathrm{Kl}_{\mathrm{c}}$, i.e., $f$ preserves the center.

Suppose that $T$ satisfies the interpolation property. It follows from [8, Theorem 2.3] that there exists a bounded distributive lattice $L$ such that $T \cong$ $\mathrm{K}(L)$, where the isomorphism is in the category $\mathrm{Kl}_{\mathrm{c}}$. Since $\mathrm{K}(L)$ satisfies $(\mathrm{CK})$, we have $T$ satisfies (CK).

Conversely, suppose that $T$ satisfies $(\mathrm{CK})$ and that $T$ does not satisfy the interpolation property. It follows from [8, Lemma 2.2] that there exist prime filters $P$ and $Q$ in $T$ and $x, y \in T$ such that $P \subseteq g(P), Q \subseteq g(Q), P \subseteq g(Q)$, and $x \wedge y \leq \sim x \vee \sim y$. We define $u=x \vee \mathrm{c}$ and $v=y \vee \mathrm{c}$, so $u, v \geq \mathrm{c}$. Straightforward computations show that $u \wedge v=c$, so it follows from condition (CK) that there exists $z \in T$ such that $z \vee \mathrm{c}=u$ and $\sim z \vee \mathrm{c}=v$. Since $u \geq x$, $v \geq y, x \in P$, and $y \in Q$, then $z \vee c \in P$ and $\sim z \vee v \in Q$. In particular, $z \in P$ or $\mathrm{c} \in P$. But $\mathrm{c}=\sim \mathrm{c}$, so $\mathrm{c} \notin P$. Hence, we have that $z \in P$. In the same way, we have that $\sim z \in Q$. But $P \subseteq g(Q)$, so we have a contradiction. Therefore, $T$ satisfies the interpolation property.

\section{REFERENCES}

[1] Balbes, R., Dwinger P.: Distributive Lattices. University of Missouri Press (1974)

[2] Bezhanishvili, N., Gehrke, M.: Finitely generated free Heyting algebras via Birkhoff duality and coalgebra. Logical Methods in Computer Science 7, 1-24 (2011)

[3] Busaniche M., Cignoli R.: Constructive Logic with Strong Negation as a Substructural Logic. Journal of Logic and Computation 20, 761-793 (2010)

[4] Castiglioni J.L., Menni M., Sagastume M.: On some categories of involutive centered residuated lattices. Studia Logica 90, 93-124 (2008)

[5] Castiglioni J.L., Lewin R., Sagastume M.: On a definition of a variety of monadic l-groups. Studia Logica 102, 67-92 (2014)

[6] Celani S.: Bounded distributive lattices with fusion and implication. Southeast Asian Bull. Math. 27, 1-10 (2003)

[7] Celani S., Jansana R.: Bounded distributive lattices with strict implication. Mathematical Logic Quarterly 51, 219-246, (2005)

[8] Cignoli R.: The class of Kleene algebras satisfying an interpolation property and Nelson algebras. Algebra Universalis 23, 262-292 (1986)

[9] Epstein G., Horn A.: Logics which are characterized by subresiduated lattices. Z. Math. Logik Grundlagen Math. 22, 199-210 (1976)

[10] Fidel M. M.: An algebraic study of a propositional system of Nelson. In: Arruda, A.I., Da Costa, N.C.A., Chuaqui, R. (eds.) Mathematical Logic. Proceedings of the First 
Brazilian Conference. Lectures in Pure and Applied Mathematics, vol. 39, pp. 99-117. Marcel Dekker, New York (1978)

[11] Kalman J.A.: Lattices with involution. Trans. Amer. Math. Soc. 87, 485-491 (1958).

[12] Sagastume M.: Kalman's construction (2007, preprint).

[13] Spinks M., Veroff R.: Constructive logic with strong negation is a substructural logic. I. Studia Logica 88, 325-348 (2008)

[14] Vakarelov, D.: Notes on $\mathcal{N}$-lattices and constructive logic with strong negation. Studia Logica 36, 109-125 (1977)

José Luis Castiglioni

Departamento de Matemática, Facultad de Ciencias Exactas, UNLP and CONICET, Casilla de correos 172, La Plata (1900), Argentina.

e-mail: jlc@mate.unlp.edu.ar

Sergio Arturo Celani

Departamento de Matemática, Facultad de Ciencias Exactas, UNCPBA and CONICET, Pinto 399, Tandil (7000), Argentina.

e-mail: scelani@exa.unicen.edu.ar

HERnán Javier SAn MarTín

Departamento de Matemática, Facultad de Ciencias Exactas, UNLP and CONICET, Casilla de correos 172, La Plata (1900), Argentina.

e-mail: hsanmartin@mate.unlp.edu.ar 